\title{
CLINICO RADIOLOGICAL STUDY OF INTERSTITIAL LUNG DISEASES AT A TERTIARY HOSPITAL
}

\author{
S. A. Rafi' , G. Sundar Raj', P. Yugandhar', K. Anil Kumar', Md. Badusha ${ }^{5}$, S. Satya Sri ${ }^{6}$
}

\author{
${ }^{1}$ Assistant Professor, Department of Pulmonary Medicine, MNR Medical College, Hyderabad. \\ ${ }^{2}$ Associate Professor, Department of Pulmonary Medicine, Asram Medical College, Eluru, Andhra Pradesh. \\ ${ }^{3}$ Professor, Department of Pulmonary Medicine, Asram Medical College, Eluru, Andhra Pradesh. \\ 4Junior Resident, Department of Pulmonary Medicine, Asram Medical College, Eluru, Andhra Pradesh. \\ $5 J u n i o r$ Resident, Department of Pulmonary Medicine, Asram Medical College, Eluru, Andhra Pradesh. \\ ${ }^{6}$ Professor and HOD, Department of Pulmonary Medicine, Asram Medical College, Eluru, Andhra Pradesh.
}

\begin{abstract}
Adult patients (34 in number) attending department of Pulmonary Medicine, ASRAM hospital with features of Interstitial lung diseases between 2009-2011 are included in this study based on thorough clinical history taking examination, serum tests, pulmonary function tests and radiological analysis.Results statistically analyzed. The present study primarily aims to diagnose interstitial lung disease with thorough clinical history, physical exam, chest radiograph, pulmonary function tests, high resolution CT scan without surgical lung biopsy. With thorough clinico radiological evaluation IPF and other IIP can be diagnosed with reasonable certainty as a part of gold standard and only atypical and indeterminate ILDs need further pathological confirmation by surgical lung biopsy.
\end{abstract}

KEYWORDS: Ild - Interstitial Lung Disease.

HOW TO CITE THIS ARTICLE: S. A. Rafi, G Sundar Raj, P. Yugandhar, K. Anil Kumar, Md. Badusha, S. Satya Sri. “Clinico Radiological Study of Interstitial Lung Diseases at a Tertiary Hospital”. Journal of Evolution of Medical and Dental Sciences 2015; Vol. 4, Issue 88, November 02; Page: 15345-15348, DOI: 10.14260/jemds/2015/2183.

INTRODUCTION: The interstitial lung diseases are a clinically challenging and diverse group of over 150 disorders characterized by varying degrees of fibrosis and inflammation of the lung parenchyma or interstitium.

The interstitial lung diseases represent many features in common such as similarities of symptoms, comparable appearance of chest imaging studies consistent alterations in pulmonary physiology and typical histological features.

However ILD are difficult to classify because approximately 150 plus known diseases are characterized by interstitial involvement, either as a primary disease or as a part of multi organ process as may occur in collagen vascular diseases one useful approach is to separate ILD into two groups, those with known or unknown causes, for each ILD there may be an acute phase followed by a phase of chronicity with acute exacerbation. ${ }^{1}$

Among ILD of known cause, the largest group includes diseases due to Inhalation of inorganic dusts, organic dusts and various irritant and noxious gases. The number of ILD of unknown cause is also very large.

The major ones are Idiopathic Pulmonary Fibrosis, Sarcoidosis, ILD associated with collagen vascular diseases. Diagnosis of ILD occurs mostly in the fifth and sixth decades, but differs between the ILD diseases, ranging from the fourth decade for sarcoidosis to the seventh decade for drugrelated ILD (Lim et al, 1996; Johnston et al, 1997;

Financial or Other, Competing Interest: None.

Submission 08-10-2015, Peer Review 09-10-2015,

Acceptance 20-10-2015, Published 02-11-2015.

Corresponding Author:

Dr. S. A. Rafi,

D. No. 612 Mahara Block, Garden Towers,

Masab Tank, Hyderabad-500028.

E-mail:drrafishaik@gmail.com

DOI:10.14260/jemds/2015/2183.
Thomeer et al, 2001a, 2001b) Current estimates of incidence and prevalence of ILD are higher than historical estimates.

The inflammation usually referred to as alveolitis is associated with spread to adjacent portions of interstitium and vasculature, resulting in derangement of alveolar capillary architecture leading to Alveolo-capillary membrane damage and loss of gas exchange units eventually ending in interstitial fibrosis. ${ }^{2}$ The scarring and distortion of lung tissue leads to deranged gas exchange $\&$ ventilator function.

When a clinician is confronted with a patient with possible ILD, in addition to a detailed medical history, physical examination and routine lab tests, a rationale approach to a diagnostic evaluation includes noninvasive diagnostic techniques such as pulmonary function tests including exercise stress test and chest imaging.

Invasive diagnostic procedures such as bronchoalveolar lavage, transbronchial lung biopsy and open lung biopsy are taken up when a specific cause of ILD remains unclear. ${ }^{3}$ In the current study profiling of the ILD on the basis of clinical and radiological certainty tried to delegate the necessity of open lung biopsy such that less trauma and associated complications avoided.

Pulmonary function tests are an important means for establishing the presence of ILD. They are also useful for the clinical monitoring of the disease course and to assess the efficacy of treatment. It is observed that a fraction of biopsy proven cases of ILD have normal plain chest films. ${ }^{4}$

Hence characteristic pulmonary function and exercise test abnormalities can suggest ILD with addition of HRCT chest coupled with BAL and TBLB (Without surgical Lung Biopsy). A restrictive pulmonary function defect and decrease in DLCO are the predominant physiological abnormalities noticed in ILD. FEV1, FVC are reduced proportionately so that the ratio of the two remains normal. 
Total lung capacity and lung volumes are reduced. PAO2-Pao2 at rest and with exercise may be normal or increased. DLco is believed to be a relatively sensitive parameter and may the only abnormality seen in early stages of ILD. The degree of arterial hypoxemia induced by exercise "A-a02" gradient correlate well with the degree of pulmonary fibrosis. Exercise test is the most sensitive diagnostic test for ILD since in some patients with biopsy proven ILDthe physiological responses to exercise are distinctly abnormal, even though base line pulmonary functions including DLco and arterial blood gases, chest radiograph and HRCT scan are all normal. ${ }^{5}$ Exercise response of ILD patients include a progressive widening of A-a 02, elevated physiological dead space,marked reduction in overall exercise tolerance and low oxygen pulse.

The mechanism of this exercise gas exchange abnormality is explained by diffusion block, across the thickened alveolar capillary membrane, low ventilation and perfusion lung units \& shunts, reduced transit time for RBC in pulmonary capillaries and a high fixed dead space due to high ventilator response for any given level of 02 consumption seen in patients with ILD Plain radiograph of the chest remains the cornerstone of the basic imaging in ILD's though in early stages the chest films can be normal. In addition, it is a good means of monitoring progression of disease and response to treatment.

It can also provide diagnostic points to possible etiologies. CT and HRCT scans are more sensitive and can detect abnormalities better than chest radiography. Preliminary studies suggest that when HRCT pattern is combined with clinical and radiological findings, it can have a diagnostic utility to increasing certainty with improved visualization and expertise.

It can also detect areas of non-fibrotic active disease and relatively unaffected areas to guide the site selection for biopsy. Broncho alveolar lavage is of diagnostic value when the specimen contains infectious agent or neoplastic cell and altered CD4: CD8 are detected. Still bone of contention. Open lung biopsy and thoracoscopic guided lung biopsy merit consideration as final diagnostic step in selected patients. Since the exact etiology and pathogenesis of most ILD are unclear, the treatment options are non specific the therapy is aimed at reducing the inflammation so that the fibrosis can be halted and the rate of progression can be slowed down. ${ }^{6}$

The clinical response is variable. Prognosis is excellent in some ILD like hypersensitivity pneumonitis when the noxious stimulus is removed .In chronic fibrotic disorders, generally the prognosis is poor. Almost half of the patients of IPF, experience subjective improvement with corticosteroid therapy and other immunosuppressive agents, but only 20 to $25 \%$ demonstrate objective improvement.

The $\mathrm{PaO} 2$ at rest and during exercise testing is superior to traditional PFT, to monitor physiologic response to therapy. Supplemental oxygen should be given to all patients with resting hypoxemia, as well those with oxy-hemoglobin desaturation with exertion. Heart and lung or single lung transplantation for highly selected patients with end stage pulmonary fibrosis is available.

Relentless progression of the disease with eventual respiratory insufficiency is inevitable and the five years survival rate is above $50 \%$. It is to be hoped that, in future major advances will be made in understanding the pathogenesis of the disorder so that novel therapeutic strategies may be invented.
In this current study Clinico-Radio logical evaluation to profile ILDS presenting to a tertiary institute done without surgical lung biopsy (both VATS \& open), TBLB/TBNA with BAL analysis done where ever necessary.

MATERIAL AND METHODS: Adult patients(34 in number) attending department of Pulmonary Medicine, ASRAM hospital with features of Interstitial lung diseases between 2009-2011 are included in this study based on thorough clinical history taking , examination, serum tests, pulmonary function tests and radiological analysis. Results statistically analyzed.

\section{Selection of the cases-Selection criteria for the study:}

Exclusion Criteria: Already diagnosed pulmonary tuberculosis /Extra pulmonary tuberculosis cases on Treatment of ATT Already diagnosed Malignancy cases. For want of facilities DLCO, Surgical lung biopsy not done for the selected cases.

Inclusion criteria: Chest symptoms -cough, progressive shortness of breath, with (or/and) wheeze, rashes, arthralgia. Radiological appearance of reticular, nodular, reticulo-nodular or/and honey combing patterns, ground glassing opacity. Sputum AFB/mantoux//Lymph node biopsy-HPE/FNAC where ever possible done to rule out Extra pulmonary tuberculosis and malignant lesions and included cases negative for the same. FOB with BAL for all cases to rule out any infectious or malignant lesions and included cases with negative report for the same.

DISCUSSION: Interstitial lung diseases are heterogeneous group of diseases involving lung interstitium. They have features in common like similarities of symptoms, comparable radiographic appearances, consistent alterations in the pulmonary physiology and typical histological features. In the present study total of 34 patients included attending ASRAM hospital during the period 2009-2011 department of Pulmonary Medicine, included etiological forms of interstitial lung diseases such as IPF, CTDCVD, occupational-pneumoconiosis etc. The main aim is to profile ILDs with clincoradiological correlation without surgical lung biopsy (both Vat and Open surgery).

In a study of patients conducted Lim G et al (Korea) the most common cause of ILD was IPF which was followed by CVD and hypersensitivity pneumonia. In present study also the most common cause is IPF accounting for $41.2 \%$ of cases followed by $23.5 \%$ were occupation \&environmental induced diseases and pneumoconiosis. Only sarcoidosis (by TBLB in some cases) showed less percentage (5.9\%) of the cases probably because of lack of yield by TBLB and confirmation by surgical lung biopsy. But most common other ILDs could be profiled conclusively in this study.

In the references to the study by Lim GI et al the sex ratio was equal in males \& females in IPF. But the female incidence was greater in sarcoidosis 131 and male incidence in pneumoconiosis. In the present study the males accountfor majority $(61.5 \%)$ in IPF. In Occupational/Pneumoconiosis also falling line with the study with $75 \%$ were males. Over all, males accounted for majority of the cases in ILD with $56 \%$ in the current study. In CVD the most common cause is Rheumatoid arthritis in Lim GI et al study, in this study also RA are most common causes in CVD. Female patients took $80 \%$ share among CVD patients showing female predominance among collagen vascular diseases in this study. 
One case of Scleroderma and one sJogrens reported in the currentstudy. Among other interstitial pneumonias one PLCH reported. In current study the characteristic radiographic (CXR) appearances observed were the Honey combing (47\%) Reticulation (25\%) Reticulonodular (29\%) with some overlap among the features taking place still consistent with the standard high ratio (47\%) among honey combing given by almost all studies. Hilar lymphadenopathy recorded in 2 cases of sarcoidosis.

HRCT chest was done in all cases. HRCT showed changes like reticulonodular pattern, traction bronchiectasis, and honey combing in UIP. Most common pattern showed is NSIP (50\%) of cases followed by UIP at close (41.2\%) catching up with the mostradiological studies representation pattern. One each case of GIP, COP, BOOP reported in the study among others.

Pulmonary function test was studied in all patients $79.6 \%$ has restrictive defect $17.6 \%$ of cases had mixed defect. There is one sporadic isolated obstructive defect probably with co-morbid COAD/COPD. Patients followed maximally after clinical evaluation in this study but $32.3 \%$ of the patients have lost follow up and who are on follow up are showing improvement with antifibrotic agents and steroids.

Two cases gone on mechanical ventilator and died and two other in due course. 55.8\% of patients are on follow up at this time with symptomatically being better.Current study has dealt all the ILD cases with thorough clinical and radiological evaluation and only where ever necessary TBLB sarcoidosis) done, and profiling of the types done. With reasonable conclusion ILD's profiled with clinicoradiological correlation in this study.

\section{BIBLIOGRAPHY:}

1. American Thoracic Society: Idiopathic pul-monary fibrosis: diagnosis and treatment. International consensus statement. American Thoracic Society (ATS), and the European Respiratory Society (ERS). Am J Respir CritCare Med 2000; 161:646-664.

2. American Thoracic Society/European Respi-ratory Society International Multidisciplinary Consensus Classification of the Idiopathic Interstitial Pneumonias: This joint statement of the American Thoracic Society (ATS), and the European Respiratory Society (ERS) was adopted by the ATS board of directors, June 2001 and by the ERS Executive Committee,June 2001. Am J Respir Crit Care Med 2002; 165:277-304.

3. Lynch JP III, Myers JL: Interstitial lung dis-eases; in Bone RC, Campbell GD Jr, Payne DK (eds): Bone's Atlas of Pulmonary and Critical Care Medicine. Baltimore, Williams \& Wilkins, 1999, pp 1-12.

4. British Thoracic Society: The diagnosis, asse-ssment and treatment of diffuse parenchymal lung disease in adults. Thorax 1999; 54(suppl 1):S1-S28.

5. Coultis DB, Zumwalt RE, Black WC, Sobonya RE: The epidemiology of interstitial lung disease. Am J Respir Crit Care Med 1994; 150: 967-972.

6. Munakata M, Asakawa M, Hamma Y, Kawakami Y: present status of idiopathic interstitial pneumonia: from epidemiology to etiology. Nihon kyobu shikkan gakkai zasshi 1994; 32 (suppl): 187-192.

\section{RESULTS:}

\begin{tabular}{|c|c|c|c|c|}
\hline \multirow{2}{*}{ Age Group } & \multicolumn{3}{|c|}{ Male } & \multicolumn{2}{c|}{ Female } \\
\cline { 2 - 5 } & $\mathbf{N}$ & Percentage & N & Percentage \\
\hline $20-29$ & 0 & 0 & 1 & 2.9 \\
\hline $30-39$ & 0 & 0 & 2 & 5.8 \\
\hline $40-49$ & 1 & 2.9 & 2 & 5.8 \\
\hline $50-59$ & 5 & 14.7 & 5 & 14.7 \\
\hline $60-69$ & 8 & 23.7 & 4 & 11.7 \\
\hline 70 and above & 2 & 5.8 & 1 & 2.9 \\
\hline \multicolumn{6}{|l}{ Table 1: Age and Sex Distribution of Study Group } \\
\hline
\end{tabular}

\begin{tabular}{|c|c|c|}
\hline Symptoms & Number & Percentage \\
\hline Dyspnoea on Exertion & 25 & 73.5 \\
\hline Cough & 14 & 41.1 \\
\hline Arthritis \& Arthralgia & 7 & 20.5 \\
\hline Skin Rashes \& Lesions & 4 & 11.7 \\
\hline Dysphagia & 1 & 2.9 \\
\hline \multicolumn{2}{|c|}{ Table 2: Symptoms Profile } \\
\hline
\end{tabular}

\begin{tabular}{|c|c|c|c|c|}
\hline \multirow{2}{*}{ Age Group } & \multicolumn{2}{|c|}{ Male } & \multicolumn{2}{c|}{ Female } \\
\cline { 2 - 5 } & $\mathbf{N}$ & Percentage & N & Percentage \\
\hline $20-29$ & 0 & 0 & 1 & 2.9 \\
\hline $30-39$ & 0 & 0 & 2 & 5.8 \\
\hline $40-49$ & 1 & 2.9 & 2 & 5.8 \\
\hline $50-59$ & 5 & 14.7 & 5 & 14.7 \\
\hline $60-69$ & 8 & 23.7 & 4 & 11.7 \\
\hline 70 and above & 2 & 5.8 & 1 & 2.9 \\
\hline \multicolumn{5}{|c|}{ Table 2: Symptoms Profile } \\
\hline
\end{tabular}




\begin{tabular}{|c|c|c|}
\hline Symptoms & Number & Percentage \\
\hline Reticular & 7 & 25 \\
\hline Reticulonodular & 5 & 29 \\
\hline Nodular & 5 & 14.7 \\
\hline Honey Combing & 16 & 47 \\
\hline Ground Glass Opacity & 4 & 11.7 \\
\hline Hailar lymphnode & 2 & 5.9 \\
\hline \multicolumn{2}{|c|}{ Table 3: Profile of CXR } \\
\hline
\end{tabular}

\begin{tabular}{|c|c|c|}
\hline Symptoms & Number & Percentage \\
\hline NSIP & 17 & 50 \\
\hline UIP & 14 & 41.2 \\
\hline GIP & 1 & 2.9 \\
\hline BOOP & 1 & 2.9 \\
\hline COP & 1 & 2.9 \\
\hline \multicolumn{3}{|c|}{ Table 4: Profile in HRCT } \\
\hline
\end{tabular}

\begin{tabular}{|c|c|c|}
\hline & No. & Percentage \\
\hline IPF & 14 & 41.2 \\
\hline Occupational pneumoconiosis & 8 & 23.5 \\
\hline CTD/CVD & 5 & 14.7 \\
\hline Others & 5 & 14.7 \\
\hline Granuloma & 2 & 5.9 \\
\hline \multicolumn{2}{|c|}{ Table 5: Diagnosis profile of ILD } \\
\hline
\end{tabular}

\begin{tabular}{|c|c|c|}
\hline Symptoms & Number & Percentage \\
\hline Restrictive & 27 & 79.6 \\
\hline Mixed & 6 & 17.6 \\
\hline Obstructive & 1 & 2.9 \\
\hline \multicolumn{3}{|c|}{ Table 6: Profile of PFT } \\
\hline
\end{tabular}

\begin{tabular}{|c|c|c|}
\hline Symptoms & Number & Percentage \\
\hline Better/Follow up & 19 & 55.8 \\
\hline Worsen/Dead & 4 & 11.7 \\
\hline Lost/No Follow up & 11 & 32.3 \\
\hline \multicolumn{2}{|c|}{ Table 7: Rofile of Clinical Course } \\
\hline
\end{tabular}

\begin{tabular}{|c|c|c|}
\hline Symptoms & Number & Percentage \\
\hline Antibiotics(Abx) & 18 & 52.9 \\
\hline Azathioprine & 5 & 14.7 \\
\hline Steroids & 15 & 44.1 \\
\hline Acetylcysteine (Acys) & 13 & 38.2 \\
\hline Perfenadone (Prf) & 13 & 38.2 \\
\hline Mechanicalvent (vent) & 2 & 5.8 \\
\hline \multicolumn{2}{|c|}{ Table 8: Profile of Therapy } \\
\hline
\end{tabular}

